\title{
Bulletin Board
}

\section{Risk of Alzheimer's disease may be affected by levels of gene in the brain}

In two studies published in PLoS One and Neurology, scientists report results that demonstrate "strong evidence that [insulin degrading enzyme] IDE is a lateonset Alzheimer disease (LOAD) gene with variants that modify risk of LOAD by influencing IDE expression.”

"In patients with

late-onset Alzheimer's disease, extracellular senile plaques develop in the brain."

Researchers have previously found an association between Alzheimer's disease and $I D E$, and these latest findings present a new angle on the role played by the gene in the disease process. Authors of the PLoS One research describe LOAD as the "most common cause of dementia in the elderly affecting approximately $10 \%$ of those over the age of 65 years."

In patients with LOAD, extracellular senile plaques develop in the brain. These plaques mainly contain aggregated amyloid- $\beta$ proteins amongst other components. IDE breaks the amyloid- $\beta$ protein apart, and investigators suggest that their research demonstrates that the level of the gene in the brain determines whether the development of Alzheimer's disease is protected against, or promoted.

Nilufer Ertekin-Taner (Mayo Clinic College of Medicine, Florida, USA) states, "we found a new mechanism of action for this Alzheimer's disease susceptibility gene, that acts by altering gene expression levels. It is an issue of the level of the normal gene, not whether a gene is in a mutant form that is not acting properly. We believe this is a novel and potentially powerful approach to understanding the complex biology behind development of Alzheimer's disease."

To carry out this research, scientists measured mRNA expression levels of
12 LOAD candidate genes. The levels were measured in the cerebella of 200 LOAD patients. Upon examination of aspects such as the gene mRNA expression levels and gene variations, the investigators identified three SNPs apparently linked with IDE expression levels. Further research led investigators to identify IDE variants in areas that are the same in humans, mice and rats. When these variants were tested for effects on gene expression, one particular genetic variation not only had the strongest effect on brain IDE but also influenced the risk of Alzheimer's disease. Different expression levels of the IDE gene was demonstrated when this variant was tested with the IDE gene in laboratory cells.

\section{"Researchers have previously found an association between Alzheimer's disease and insulin-degrading enzyme (IDE)..."}

Ertekin-Taner suggests that in the future, the methods of testing genes for their effects on disease risk and gene expression levels should be utilized to identify Alzheimer's disease susceptibility genes. He states, "we show that measuring gene expression leads us to disease susceptibility genes in a way that is more powerful that just looking for genetic variations that influence disease risk. There is no way we would have found this IDE variant if we only looked for its effect on disease risk and not disease expression."

Sources: Carrasquillo MM, Belbin O, Zou F et al: Concordant association of insulin degrading enzyme gene (IDE) variants with IDE mRNA, $A \beta$, and Alzheimer's disease. PLoS One 5(1), E8764 (2010); Zou F, Carrasquillo MM, Pankratz VS et al.: Gene expression levels as endophenotypes in genome-wide association studies of Alzheimer disease. Neurology 74(6), 480-486 (2010).

\section{in the news...}

- Lead story: Risk of Alzheimer's disease may be affected by levels of gene in the brain

- Large-scale trial suggests ethosuximide as best treatment for absence epilepsy pg 350

- Priority Paper Alerts pg 350

- Does age have a role in schizophrenia? pg 351

- Genome sequencing reveals Charcot-Marie-Tooth disease mutation pg 351 


\section{Large-scale trial suggests ethosuximide as best treatment for absence epilepsy}

To date, there has been no definitive evidence on the best treatment for childhood absence epilepsy, but a recent large-scale, multicenter study conducted as part of the NIH Childhood Epilepsy Study Group has suggested that ethosuximide is the most suitable drug treatment, both in terms of efficacy and tolerability.

Absence epilepsy is the most common form of pediatric epilepsy syndrome and up to $17 \%$ of childhood epilepsies are of this type. Nonconvulsive seizures are a feature of the condition, where the child will stop what they are doing and stare for up to $30 \mathrm{~s}$. The condition is thought to interfere with the child's ability to learn and play and so the importance of finding an effective treatment for this condition is apparent.

"Much of our scientific understanding of childhood epilepsy care today comes from historical experience or studies involving adult patients with related, but not identical, conditions," explains Colin Roberts, Director of the Pediatric Epilepsy Program at Oregon Heath \& Science University (OHSU) Doernbecher Children's Hospital and principal investigator of the recent study. The double-blind, controlled trial, published in New England Journal of Medicine, aimed to establish the best treatment for children with the disorder.

The study was conducted in 32 comprehensive pediatric epilepsy centers across the USA. A total of 453 children newly diagnosed with absence epilepsy were enrolled between 2004 and 2007. The patients were then randomly assigned to one of three pharmacological treatments; ethosuximide, valproic acid or lamotrigine. The dosage was incrementally increased until the child was free of seizures, the maximal tolerable dose was reached, or a criterion indicating treatment failure was met. After 16 weeks of therapy, the researchers compared the efficacy, tolerability and neuropsychological effects of the three drugs.

It was found that valproic acid and ethosuximide were significantly more effective than lamotrigine in controlling seizures. No significant differences were found among the three drugs in terms of discontinuation because of adverse effects but attentional dysfunction was more common with valproic acid than with ethosuximide.
The authors suggest that the findings of the study are of great importance. Roberts comments, "this study is an important milestone in our understanding of childhood absence epilepsy. Never before have we been able to document in such a comprehensive, scientific fashion the best options to treat children with this condition."

The researchers also suggest that largescale, multicenter studies have great potential for further research into childhood epilepsy. Roberts concludes, "the initial outcomes from this study describe one of many aspects of childhood absence epilepsy evaluated by the study group. Collaborative studies like this lay the groundwork for many critically important studies to follow that will define the proper care of children with seizures."

Sources: Oregon Health \& Science University www. ohsu.edu/xd/about/news_events/news/new-studyidentifies-best-trea.cfm; Glauser TA, Cnaan A, Shinnar S: Childhood Absence Epilepsy Study Group: ethosuximide, valproic acid, and lamotrigine in childhood absence epilepsy. N. Engl. J. Med. 362(9), 790-799 (2010).

\section{Priority Paper Alerts}

Dopamine D2 receptors in addiction-like reward dysfunction and compulsive eating in obese rats. Johnson PM, Kenny PJ: Nat. Neurosci. DOI:10.1038/nn.2519 (2010) (Epub ahead of print).

The development of obesity was found to be associated with a decrease in neural reward responses. These changes were similar to
those seen in the transition from recreational to addictive drug-taking. By measuring food consumption in spite of an aversive condi-
tioned stimulus, it was demonstrated that obese but not lean rats showed compulsive-like feeding behaviors. Similar to in drug-addicted
humans, striatal dopamine D2 receptors were downregulated in obese rats. It was also shown that knockdown of dopamine D2 receptors
speeded up the development of neural reward deficits and the onset of compulsive eating. It is suggested that the overconsumption
of food causes neuroadaptive responses in reward circuits that are like those observed in drug addiction. It is therefore suggested that
common hedonic mechanisms may underlie both drug addiction and obesity.
Tooth pulp inflammation increases brain-derived neurotrophic factor expression in rodent trigeminal ganglion neurons. Tarsa L,
Batkowiec-Iskra E, Kratochvil FJ 3rd et al.: Neuroscience DOI: 10.1016/j.neuroscience.2010.03.002 (2010) (Epub ahead of print).
The mechanisms of chronic inflammatory pain are yet to be fully understood. Previous research identified neurotrophin brain-derived
neurotrophic factor (BDNF) as a protein expressed by rat trigeminal ganglion (TG) neurons by electrical stimulation and calcitonin
gene-related peptide (CGRP), which is a mediator of trigeminal inflammatory pain. This study aimed to investigate the effects of inflam-
mation on BDNF expression in TG neurons of juvenile rats and mice. Tooth pulp inflammation was used as a model. Cavities were made
in the teeth on the right side of the mouth of the animals and were then left open to oral microflora. The expression level of BDNF in the
TG neurons on the right side of the mouth was compared with that on the left side of the same animal. It was confirmed that exposing
the tooth pulp for 28 days, with consequent inflammation, leads to a significant upregulation of BDNF in the TG close to the affected
teeth. It is suggested that mouse TG provides a suitable model to study in vivo molecular mechanisms of inflammation-dependent
regulation of BDNF expression. 


\section{Does age have a role in schizophrenia?}

A recent study has highlighted new data on how gene regulation and age can play a role in schizophrenia.

The researchers from Scripps Research Institute (CA, USA) performed integrated gene coexpression network analysis on two large microarray-based brain gene expression data sets generated from the prefrontal cortex'. These data sets were taken post-mortem from a total of 101 participants; 54 normal control subjects and 47 schizophrenia sufferers.

Genes were not just examined individually, but in order to find dysfunctional cellular pathways in schizophrenia, gene interactions were also considered; along with groups of genes that demonstrated similar expression patterns. Elizabeth Thomas (Scripps Research Institute, CA, USA) states, "once gene co-expression networks are identified, we can then ask how they are affected by factors such as age or drug treatment, or if they are associated with particular cell types in the brain."

One specific finding of this research, published in Genome Research, was a "robust effect of age on gene expression modules that differentiates normal subjects from those with schizophrenia." The group found that thirty genes associated with developmental processes of the nervous system, such as neuronal differentiation and neurite outgrowth, were turned on in schizophrenia patients, whereas these genes should normally become inactive as a person ages.

Authors of the study explain that, "extrapolating these findings to earlier stages of development supports the concept that schizophrenia pathogenesis begins early in life and is associated with a failure of normal decreases in developmental-related gene expression." Thomas states that, "rather than a pathological trigger occurring at a critical developmental time point, the trigger is ongoing throughout development and aging." The authors suggest that approaches to the treatment of schizophrenia could be changed owing to these results and, according to Thomas, this study supports "early intervention and treatment of schizophrenia."

Sources: Torkamani A, Dean B, Schork NJ, Thomas EA: Coexpression network analysis of neural tissue reveals perturbations in developmental processes in schizophrenia. Genome Res. DOI:10.1101/gr.101956.109 (2010) (Epub ahead of print).

\section{Genome sequencing reveals Charcot-Marie-Tooth disease mutation}

The causative mutation associated with the nerve function disorder, Charcot-Marie-Tooth disease (CMT) has been identified by the whole-genome sequencing of one of its sufferers. The researchers responsible for this new discovery hope that it may lead to a more effective treatment for the disorder.

James R Lupski of the Baylor College of Medicine (TX, USA) is a doctor and a geneticist, but also a sufferer of CMT. Lupski and his colleague Richard Gibbs, also of the Baylor College of Medicine, worked with Life Technologies Corp. (CA, USA), using next-generation techniques to identify the mutation that causes CMT.

Charcot-Marie-Tooth disease is one of the more common neurological disorders and it affects one in 2500 individuals in the USA. The condition mainly affects the peripheral nerves and patients may have weak lower limb muscles, which can affect gait or cause difficulty in fine motor control of the hands.
The researchers sequenced the whole of Lupski's genome and utilized a ultra-highthroughput next-generation genomic analysis platform in order to identify the causal genetic variant in CMT. Lupski, who is one of three CMT sufferers in his immediate family, has been researching the disease for over 20 years and has high hopes for the implications of this discovery. He explains, "I've dedicated most of my career to understanding the genetic basis of CMT for very personal reasons. By having a better understanding of this disease, my hope is that we - the healthcare industry and individuals affected by CMT - will be able to better mitigate its consequences."

"...Charcot-Marie-Tooth disease (CMT) has been identified by the whole-genome sequencing of one of its sufferers."

The authors of the study, published in New England Journal of Medicine, claim that this is the first time whole-genome sequencing has been used to identify the cause of an inherited disease. Shaf Yousaf, President of Genomic Analysis for Life Technologies, explains how this can translate to research in other areas. He states, "the elucidation of this genetic mutation using high-throughput sequencing is a powerful statement about the use of advanced genome analysis technologies as a discovery and diagnostic research tool and will help accelerate the promise of personalized medicine." He continues, "there is tremendous opportunity to apply this research as proof-of-principle and rapidly advance the discovery of other rare genetic variants associated with disease."

Sources: Life Technologies Corporation (CA, USA) www.lifetechnologies.com/scientists-discovercausative-genetic-mutation-associated-commoninherited-neurological-disorder.ht-0; Lupski JR, Reid JG, Gonzaga-Jauregui C et al.: Whole-genome sequencing in a patient with Charcot-Marietooth neuropathy. N. Engl. J. Med. DOI:10.1056/ NEJMoa0908094 (2010) (Epub ahead of print). 